\title{
Uneven HAK/KUP/KT Protein Diversity Among Angiosperms: Species Distribution and Perspectives
}

\author{
Manuel Nieves-Cordones ${ }^{*}$, Reyes Ródenas ${ }^{2}$, Alain Chavanieu ${ }^{3}$, Rosa M. Rivero ${ }^{2}$, \\ Vicente Martinez ${ }^{2}$, Isabelle Gaillard ${ }^{1}$ and Francisco Rubio ${ }^{*}$
}

${ }^{1}$ Biochimie et Physiologie Moléculaire des Plantes, Institut de Biologie Intégrative des Plantes, UMR 5004 CNRS/UMR 0386 INRA/Montpellier SupAgro/Université Montpellier 2, Montpellier, France, ${ }^{2}$ Department of Plant Nutrition, Centro de Edafología y Biología Aplicada del Segura, Consejo Superior de Investigaciones Científicas, Murcia, Spain, ${ }^{3}$ Institut des Biomolécules Max Mousseron, UMR 5247, Faculté de Pharmacie, Montpellier, France

\section{OPEN ACCESS}

Edited by:

Manuel González-Guerrero, Universidad Politécnica de Madrid,

Spain

Reviewed by:

Ingo Dreyer,

Universidad de Talca, Chile

Ryoung Shin,

RIKEN Center for Sustainable

Resource Science, Japan

*Correspondence:

Manuel Nieves-Cordones nieves@supagro.inra.fr;

Francisco Rubio

frubio@cebas.csic.es

Specialty section:

This article was submitted to

Plant Nutrition,

a section of the journal

Frontiers in Plant Science

Received: 03 December 2015

Accepted: 23 January 2016

Published: 09 February 2016

Citation:

Nieves-Cordones $M$, Ródenas $R$, Chavanieu A, Rivero RM, Martinez V, Gaillard I and Rubio F (2016) Uneven HAK/KUP/KT Protein Diversity Among Angiosperms: Species Distribution and Perspectives. Front. Plant Sci. 7:127. doi: 10.3389/fpls.2016.00127
$\mathrm{HAK} / \mathrm{KUP} / \mathrm{KT} \mathrm{K}^{+}$transporters have been widely associated with $\mathrm{K}^{+}$transport across membranes in bacteria, fungi, and plants. Indeed some members of the plant HAK/KUP/KT family contribute to root $\mathrm{K}^{+}$uptake, notably at low external concentrations. Besides such role in acquisition, several studies carried out in Arabidopsis have shown that other members are also involved in developmental processes. With the publication of new plant genomes, a growing interest on plant species other than Arabidopsis has become evident. In order to understand HAK/KUP/KT diversity in these new plant genomes, we discuss the evolutionary trends of $913 \mathrm{HAK} / \mathrm{KUP} / \mathrm{KT}$ sequences identified in 46 genomes revealing five major groups with an uneven distribution among angiosperms, notably between dicotyledonous and monocotyledonous species. This information evidenced the richness of crop genomes in HAK/KUP/KT transporters and supports their study for unraveling novel physiological roles of such transporters in plants.

Keywords: HAK/KUP/KT, transporter, potassium, phylogeny, angiosperm

\section{INTRODUCTION}

Potassium is an essential macronutrient for plants, making up to $2-7 \%$ of the plant's total dry weight (Evans and Sorger, 1966; Leigh and Wyn Jones, 1984). It fulfills a number of important functions, such as enzyme activation, neutralization of negative charges and, more specific to plants, the maintenance of cell turgor that leads to plant growth and organ movement (Marschner, 2012). As sessile organisms, plants need to take up $\mathrm{K}^{+}$from the soil. This is firstly achieved by root epidermal and cortical cells. Then, $\mathrm{K}^{+}$is loaded in the stele and transported to the shoot and distributed to the leaves (Ahmad and Maathuis, 2014; Wigoda et al., 2014). Potassium shortand long-distance transport involves the movement of $\mathrm{K}^{+}$through cell membranes, notably the plasma membrane which in many cases occurs against steep concentration gradients (like in the root-soil interface, for instance). In plants, there are five major multi-gene families that encode $\mathrm{K}^{+}$permeable transport systems: (i) Shaker-like $\mathrm{K}^{+}$channels, (ii) tandem-pore $\mathrm{K}^{+}(\mathrm{TPK})$ channels, (iii) HAK/KUP/KT transporters, (iv) HKT transporters, and (v) cation-proton antiporters (CPAs; Mäser et al., 2001). They have become the essentials of the $\mathrm{K}^{+}$transport toolkit during terrestrial plant evolution due to their widespread presence in different land plant lineages (Gomez-Porras et al., 2012). 
Here we focus on the HAK/KUP/KT (High-Affinity $\mathrm{K}^{+} / \mathrm{K}^{+}$UPtake $/ \mathrm{K}^{+}$Transporter) transporter family. Plant $\mathrm{HAK} / \mathrm{KUP} / \mathrm{KT}$ transporters were first identified in barley and Arabidopsis (Quintero and Blatt, 1997; Santa-María et al., 1997; Fu and Luan, 1998; Kim et al., 1998) from their homology to bacterial KUP and fungal HAK transporters (Schleyer and Bakker, 1993; Bañuelos et al., 1995). Due to the different acronyms used in these early reports, the composite name of HAK/KUP/KT is widely used to refer to the whole family in plants. Plant HAK/KUP/KT proteins possess 10-15 transmembrane (TM) segments with both $\mathrm{N}$ - and C-termini in the intracellular side of the membrane, the latter being much longer (Rubio et al., 2000; Gomez-Porras et al., 2012). They have been widely shown to mediate $\mathrm{K}^{+}$fluxes when expressed in $\mathrm{K}^{+}$uptake deficient bacteria or yeast. Moreover, plant HAK/KUP/KT proteins differ in their affinity for $\mathrm{K}^{+}$and can mediate cation influx as well as efflux (Fu and Luan, 1998; Rubio et al., 2000; Senn et al., 2001; Bañuelos et al., 2002; Garciadeblas et al., 2002; Ahn et al., 2004). Different studies reported that HAK/KUP/KT transporters poorly discriminate between $\mathrm{K}^{+}, \mathrm{Rb}^{+}$, and $\mathrm{Cs}^{+}$ and are inhibited by $\mathrm{NH}_{4}{ }^{+}$(Santa-María et al., 1997; Rubio et al., 2000; Bañuelos et al., 2002; Martínez-Cordero et al., 2004). Plant HAK/KUP/KT proteins exhibit a great diversity in terms of subcellular localization (plasma membrane, tonoplast, or other endomembranes; Bañuelos et al., 2002; Jaquinod et al., 2007; Qi et al., 2008; Osakabe et al., 2013; Rigas et al., 2013) and expression patterns (root meristems, vascular tissues, guard cells, fruits, or specialized organs such as flytraps; Elumalai et al., 2002; Ahn et al., 2004; Vicente-Agullo et al., 2004; Davies et al., 2006; Osakabe et al., 2013; Scherzer et al., 2015).

Regarding their functions, some members of the plant HAK/KUP/KT family contribute to root $\mathrm{K}^{+}$uptake, notably at low external concentrations (high-affinity range) through active $\mathrm{K}^{+}$transport (Nieves-Cordones et al., 2014). Such highaffinity $\mathrm{K}^{+}$transporters are expected to be $\mathrm{H}^{+} / \mathrm{K}^{+}$symporters (Rodriguez-Navarro et al., 1986; Maathuis and Sanders, 1994), but experimental support for this notion is still required. Several studies carried out in Arabidopsis have shown that other members are involved in the regulation of cell size, auxin distribution or osmotic stress adaptation (Very et al., 2014). Such three roles highlight the great importance and role diversity of HAK/KUP/KT transporters in plant physiology besides $\mathrm{K}^{+}$ acquisition.

During the last two decades, research on Arabidopsis has notably accelerated the acquisition of information concerning the molecular and physiological mechanisms around $\mathrm{K}^{+}$transport and HAK/KUP/KT proteins. This has been possible mainly because of the availability of its genome sequence and the use of T-DNA insertion lines to knock-out gene function. In recent years, genome sequences from many plant species have become available. This, together with the establishment of genomeediting techniques, such as Transcription Activator-Like Effector Nucleases (TALEN) or Clustered Regularly Interspaced Short Palindromic Repeats-Cas system (CRISPR-Cas) opens the door to investigate $\mathrm{HAK} / \mathrm{KUP} / \mathrm{KT}$ gene function in crops much faster (Andersen et al., 2015). It is true that research on crop species can benefit from the information gained in Arabidopsis, but the study of certain physiological processes, such as the development of a fleshy fruit, need to be carried out in appropriate species. In order to orientate further research in HAK/KUP/KT function in crop species, we present a multi-species phylogenetic analysis of plant HAK/KUP/KT proteins (comprising 913 members from 46 sequenced genomes) evidencing the presence of five major clades and remarkable specificities depending on the angiosperm group considered.

\section{HAK/KUP/KT PHYLOGENY IN ANGIOSPERMS}

Phylogenetic relationships within the HAK/KUP/KT family have consistently shown the existence of several clades in angiosperm species, but with weak biological support for such distribution (Rubio et al., 2000; Gupta et al., 2008; Gomez-Porras et al., 2012; Very et al., 2014). Since the number of sequenced angiosperm genomes, and thus that of HAK/KUP/KT available sequences, has notably increased in the last years, we wanted to assess the robustness and the species distribution of the different clades. For that purpose, we made an inventory of HAK/KUP/KT protein sequences from 43 angiosperm genomes plus three outgroup species (one gymnosperm, Picea abies, and two primitive nonseed plants Selaginella moellendorffii and Physcomitrella patens; Supplementary Table S1). The phylogenetic tree obtained by maximum likelihood for such sequences revealed five major clades (I to V) where I to IV followed previous numeration (Rubio et al., 2000; Gupta et al., 2008; Gomez-Porras et al., 2012; Very et al., 2014) (Figure 1). Representative HAK/KUP/KT transporters that have been functionally characterized are found throughout the tree. Several subgroups were identified in clade I ( $\mathrm{Ia}$ and Ib) and in clade II (IIa, IIb, and IIc). Then, we assessed the HAK/KUP/KT sequence distribution in the different analyzed species and the angiosperm orders to which they belong (Table 1). Results from the common ancestor of dicotyledonous and monocotyledonous species, Amborella trichopoda, evidenced the presence of HAK/KUP/KT transporters in that ancestor in all of the aforementioned clades. They also suggested that clade I separation into Ia and $\mathrm{Ib}$ occurred at the beginning of the angiosperm lineage since A. trichopoda has Ia and Ib transporters and outgroup sequences belonging to clade I were not placed in any of this two major subclades. It is worth to note that clade Ib only contained sequences from dicotyledonous species, but not from monocotyledonous ones (Figure 1, Table 1). This result suggests that clade $\mathrm{Ib}$ disappeared in the monocotyledonous lineage because, as stated before, it was already present in the A. trichopoda genome. Within HAK/KUP/KT transporters from dicotyledonous genomes, different transporter distributions among orders were identified and, in some cases, groups of related species displayed empty clades that are indicative of important events in the evolution of HAK/KUP/KT transporters in dicotyledonous orders. Indeed, in Solanales, clade IIb transporters were not identified, while in Cucurbitales it was the case for clade IV. The analysis of HAK/KUP/KT transporters from Brassicales species provided striking results: clades Ib and IV were absent in HAK/KUP/KT transporters from 


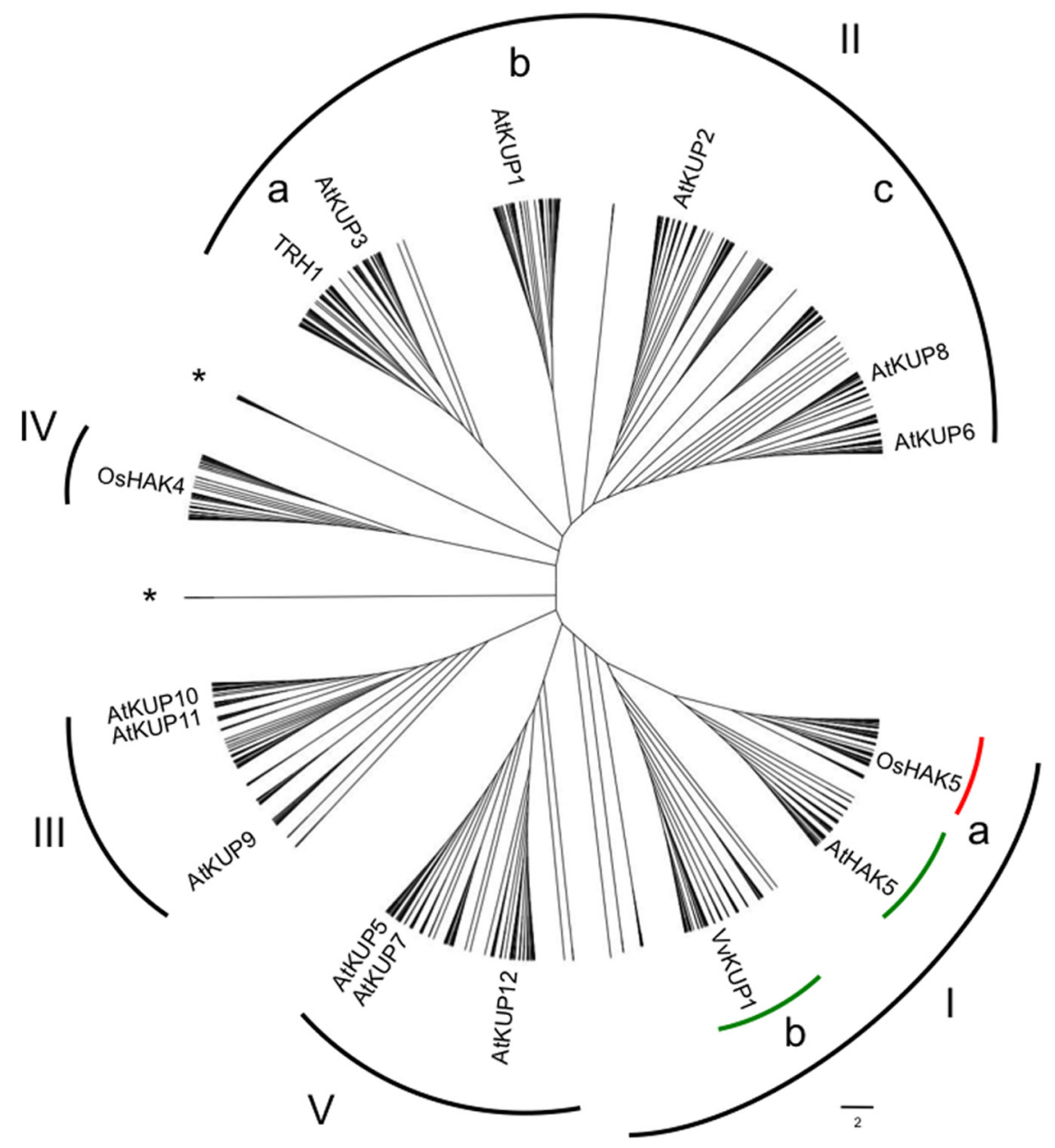

FIGURE 1 | Phylogenetic tree of the HAK/KUP/KT family in plants containing 913 sequences from 46 fully sequenced angiosperm genomes plus three outgroups (Picea abies, Selaginella moellendorffii, and Physcomitrella patens). Protein sequences fall into five main clades (I to V) where $V$ is a novel clade. Some sub-clades within clade I contain only sequences from dicotyledonous (green lines) or monocotyledonous (red line) species. Asterisks represent outgroup sequences, which did not fall into main clades. Letters depict sub-clades within clades I and II. Representative members within the different clades are shown. Retrieved sequences from public genomic resources were ascribed to the HAK/KUP/KT family by using Orthomcl (http://orthomcl.org/orthomcl/). Protein organization was evaluated with MEME suite website (http://meme-suite.org/). Then, sequences were aligned by MAFFT (http://mafft.cbrc.jp/alignment/server/) and then alignment curation by G-block analysis in Seaview was applied prior to tree building. Tree building was constructed with MEGA6 by maximum-likelihood analysis. The scale bar represents number of substitutions per site. See also Supplementary Table S1.

the Brassicaceae family (eight genomes, including Arabidopsis thaliana) while in Carica papaya (belonging to Brassicales but not to such family) had one transporter belonging to clade Ib and three in clade IV. Thus, a loss of both clades could have taken place during the evolution of Brassicaceae. At the outgroup level, HAK/KUP/KT transporters from Physcomitrella patens were found in clade I, IV, and V whereas 13 sequences from this organism fell apart in two separate branches independent from the five major clades. With respect to Selaginella moellendorffi and Picea abies, we did not observe sequences in clades IIa and IIb in the former and in clade III in the latter.

Despite the number of HAK/KUP/KT transporters whose physiological role has been established is relatively small, some conclusions can be drawn from the present analysis.
Transporters involved in root high-affinity $\mathrm{K}^{+}$uptake both from dicotyledonous or monocotyledonous species fall into clade Ia: HvHAK1, AtHAK5, OsHAK1, CaHAK1, SlHAK5/LeHAK5, and EsHAK5/ThHAK5, for instance (Santa-María et al., 1997; Bañuelos et al., 2002; Martínez-Cordero et al., 2004; NievesCordones et al., 2007; Rubio et al., 2008; Aleman et al., 2009). However, recent work on rice OsHAK5 and OsHAK21, which also belong to clade Ia, showed more specialized functions when compared to the typical high-affinity $\mathrm{K}^{+}$transporter OsHAK1 (Chen et al., 2015). For instance, OsHAK5 and OsHAK21 were involved in $\mathrm{K}^{+}$transport to aerial parts during $\mathrm{K}^{+}$deficiency or salt stress, respectively (Yang et al., 2014; Shen et al., 2015). Since rice and other grasses belonging to the Poaceae family exhibited a higher number of clade Ia HAK/KUP/KT sequences than dicotyledonous genomes (10.29 vs. 1.84$)$, it could 
TABLE 1 | HAK/KUP/KT gene distribution among angiosperm orders and outrgroups.

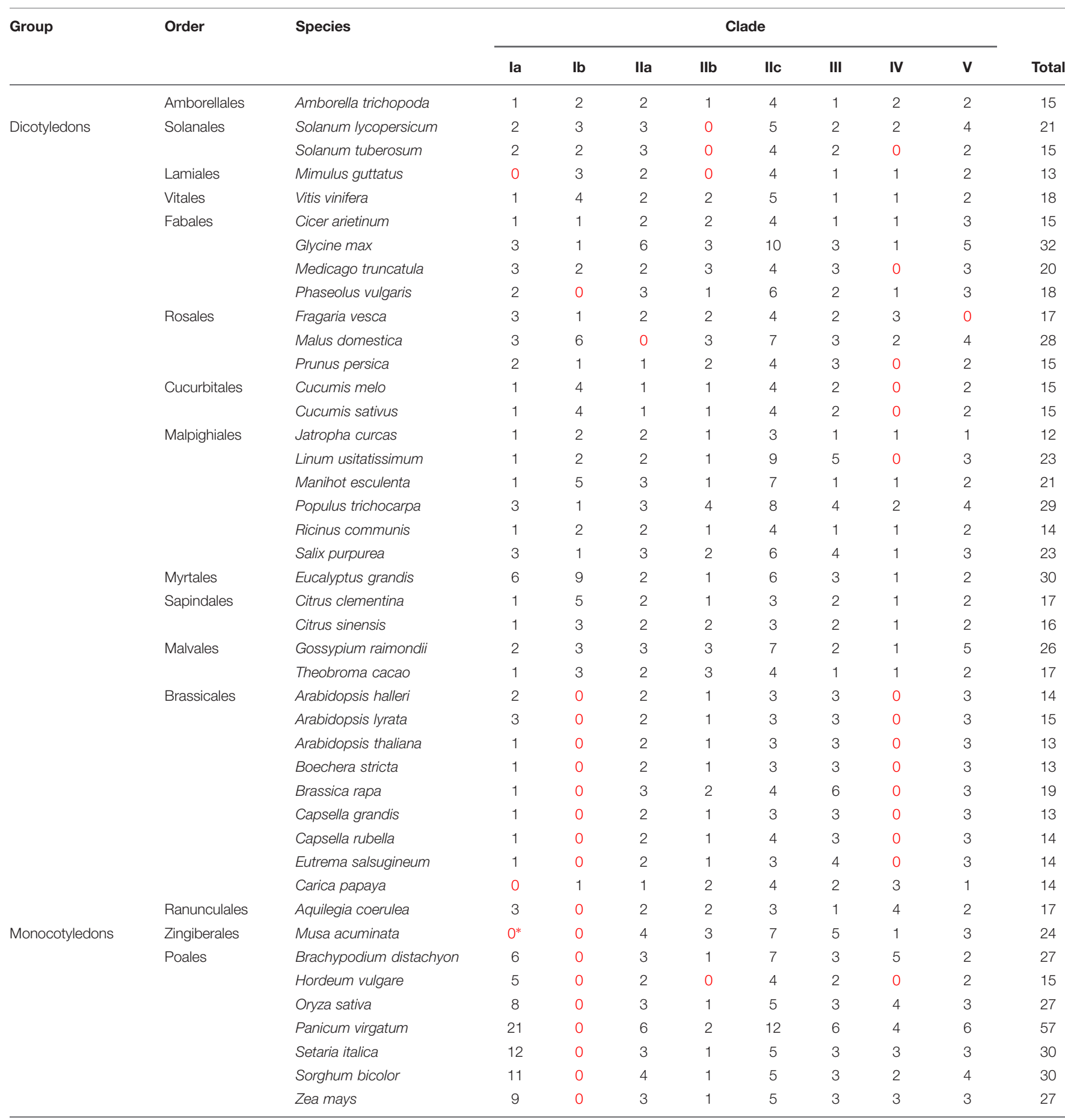

Outgroups

Gymnosperms
Lycopodiophytes
Bryophytes

Species
Cluster

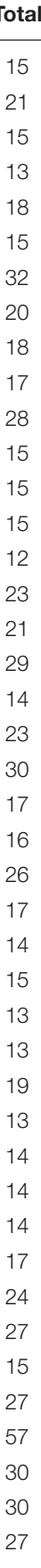

21 23 
be interpreted as a specific diversification of Ia high-affinity $\mathrm{K}^{+}$transporters in Poaceae species. It is worth to note that disruption of the OsHAK1 gene led to a dramatic decrease in grain yield (Chen et al., 2015), whereas such a phenotype has not been observed in the AtHAK5 KO mutant (Nieves-Cordones and Rubio, unpublished results). It would be interesting to know which is the contribution to grain yield of OsHAK1like transporters from other cereal species, since they could be good targets to improve food production. Regarding clade $\mathrm{Ib}$ $\mathrm{HAK} / \mathrm{KUP} / \mathrm{KT}$ transporters, two reports have provided us with some information about this group. First, VvKUP1/VvHAK1a from grapevine was shown to be expressed in flowers and grape berry skin, showing its highest expression level in the latter tissue during the pre-veraison stage (Davies et al., 2006). Second, DmHAK5 from Dionaea muscipula (Venus flytrap) contributes to high-affinity $\mathrm{K}^{+}$uptake in digesting traps (Scherzer et al., 2015). Further characterization of clade Ib transporters will clarify whether they are specialized in transporting $\mathrm{K}^{+}$in tissues other than roots. Interestingly, recent reports showed that some clade I HAK/KUP/KT transporters, including DmHAK5 (clade Ib), SlHAK5, CaHAK1, and AtHAK5 (clade Ia), are activated by CIPK23-CBL1/9 complexes, which provide novel insights into the regulation of high-affinity $\mathrm{K}^{+}$transport (Ragel et al., 2015; Scherzer et al., 2015). Moreover, such regulatory network offers a new alternative that could be used to enhance $\mathrm{K}^{+}$uptake in tomato and pepper plants.

Clade II has been associated in Arabidopsis with developmental processes, especially those which demand turgordriven cell expansion. In clade IIa, there is AtKUP4/TRH1 (Tiny Root Hairs 1) which contributes to the polar localization of auxin transporters in the root apex that, in turn, establishes auxin gradients necessary for both gravitropic responses and root hair formation (Rigas et al., 2001, 2013; Vicente-Agullo et al., 2004). The first cloned HAK/KUP/KT transporter from Arabidopsis, AtKUP1/KT1, belongs to clade IIb, but no physiological role has been attributed to it so far (Quintero and Blatt, 1997; Fu and Luan, 1998; Kim et al., 1998). In clade IIc, there are AtKUP2/6/8 which have been shown to negatively regulate plant growth and cell size by mediating $\mathrm{K}^{+}$efflux rather influx (Osakabe et al., 2013). Analysis of an AtKUP2/6/8 triple null mutant also evidenced impaired ABA responses in guard cells and lateral root cells. Phosphorylation of AtKUP6 by OST1 connected osmotic stress adaptation to the regulation of $\mathrm{K}^{+}$fluxes mediated by $\mathrm{HAK} / \mathrm{KUP} / \mathrm{KT}$ transporters.

With respect the other clades, GhKT1 from cotton (clade III; Gossypium hirsutum) was specifically upregulated during cotton fiber elongation (Ruan et al., 2001). Regarding clade IV transporters, only two have been characterized so far. LjKUP from Lotus japonicus was highly expressed during late nodulation development and complemented $\mathrm{K}^{+}$uptake deficient bacteria (Desbrosses et al., 2004). On the other hand, PpHAK13 from the outgroup species Physcomitrella patens is a high-affinity $\mathrm{Na}^{+}$ transporter, with low $\mathrm{K}^{+}$permeability, that was repressed under the presence of high $\mathrm{Na}^{+}$concentrations (Benito et al., 2012). The latter transporter raises the question whether other plant HAK/KUP/KT transporters are permeable to $\mathrm{Na}^{+}$at low external concentrations. Finally, belonging to clade V, PpHAK1 from
Physcomitrella patens was shown to regulate steady $\mathrm{K}^{+}$content and plant morphology under non- $\mathrm{K}^{+}$-limiting conditions and to contribute to high-affinity $\mathrm{Rb}^{+}$and $\mathrm{Cs}^{+}$uptake during $\mathrm{K}^{+}$ starvation (Garciadeblas et al., 2007).

Besides their physiological roles, subcellular localization of $\mathrm{HAK} / \mathrm{KUP} / \mathrm{KT}$ transporters has been assessed in some cases and it was shown to be quite diverse. Furthermore, there is not a clear relationship between phylogenetic clade to which a transporter belongs and its targeted cell membrane. For instance, several members are targeted to the plasma membrane, such as AtHAK5, OsHAK1, OsHAK21, OsHAK5 (clade I; Qi et al., 2008; Yang et al., 2014; Chen et al., 2015; Shen et al., 2015), AtKUP6 (clade IIc; Osakabe et al., 2013), and LjKUP (clade IV; Desbrosses et al., 2004) while others are targeted to the tonoplast (OsHAK10, clade IIc, and AtKUP5 clade V; Jaquinod et al., 2007; Bañuelos et al., 2002) or endoplasmatic reticulum-like endomembranes (AtKUP4/TRH1; Rigas et al., 2013).

\section{CONCLUSION AND PERSPECTIVES}

Plant $\mathrm{HAK} / \mathrm{KUP} / \mathrm{KT} \mathrm{K}^{+}$transporters have been shown to play key roles in plant physiology like $\mathrm{K}^{+}$acquisition, abiotic stress adaptation and developmental processes. Interestingly, the fact that $\mathrm{HAK} / \mathrm{KUP} / \mathrm{KT}$ transporters are permeable to $\mathrm{K}^{+}$ only explains a part of the phenotypes exhibited by the plants lacking them as it is the case of AtHAK5 or AtKUP4/TRH1, where energization of AtHAK5-mediated $\mathrm{K}^{+}$uptake or the relationship between auxin distribution and AtKUP4/TRH1 activity deserve further attention. From our analysis, it can be deduced that the contribution of $\mathrm{HAK} / \mathrm{KUP} / \mathrm{KT} \mathrm{K}^{+}$transporters to plant physiology may substantially differ among species, especially when entire clades are missing in a given group of species as shown. Therefore, Arabidopsis can still be a good model for certain well conserved roles of $\mathrm{HAK} / \mathrm{KUP} / \mathrm{KT} \mathrm{K}^{+}$ transporters, AtHAK5 for example, but research on other species, notably crops, is required: (i) to study transporters belonging to clades Ib or IV (missing in the Brassicaceae family) or clades where significant gene duplication occurred (clade Ia in monocots) and (ii) to investigate physiological aspects which are absent in Arabidopsis (fleshy fruit development, for instance). Besides, some HAK/KUP/KT proteins can be already regarded as interesting candidates for future crop improvement strategies, for example GhKT1 (specifically upregulated during cotton fiber elongation), VvKUP1/VvHAK1-a (highly expressed during the preveraison stage) and OsHAK1 (critical for rice grain yield). In line with this statement, the peach fruit is the organ where more HAK/KUP/KT genes are expressed in this species (Song et al., 2015). Further research on this transporter family will contribute to understanding how we can engineer plants for food and renewable biomass production.

\section{AUTHOR CONTRIBUTIONS}

$\mathrm{MN}-\mathrm{C}, \mathrm{RR}$, and FR performed the experimental analyses. MN-C wrote the article with inputs from AC, RMR, VM, IG, and FR. 


\section{FUNDING}

This work was funded by grant AGL2012-33504 from Ministerio de Economia y Competitividad, Spain. RR is recipient of an FPU predoctoral contract from Ministerio de Educación, Cultura y Deporte, Spain.

\section{REFERENCES}

Ahmad, I., and Maathuis, F. J. (2014). Cellular and tissue distribution of potassium: physiological relevance, mechanisms and regulation. J. Plant Physiol. 171, 708-714. doi: 10.1016/j.jplph.2013.10.016

Ahn, S. J., Shin, R., and Schachtman, D. P. (2004). Expression of KT/KUP genes in Arabidopsis and the role of root hairs in $\mathrm{K}^{+}$uptake. Plant Physiol. 134, 1135-1145. doi: 10.1104/pp.103.034660

Aleman, F., Nieves-Cordones, M., Martinez, V., and Rubio, F. (2009). Differential regulation of the HAK5 genes encoding the high-affinity $\mathrm{K}^{+}$transporters of Thellungiella halophila and Arabidopsis thaliana. Environ. Exp. Bot. 65, 263-269. doi: 10.1016/j.envexpbot.2008.09.011

Andersen, M. M., Landes, X., Xiang, W., Anyshchenko, A., Falhof, J., Østerberg, J. T., et al. (2015). Feasibility of new breeding techniques for organic farming. Trends Plant Sci. 20, 426-434. doi: 10.1016/j.tplants.2015.04.011

Bañuelos, M. A., Garciadeblas, B., Cubero, B., and Rodríguez-Navarro, A. (2002). Inventory and functional characterization of the HAK potassium transporters of rice. Plant Physiol. 130, 784-795. doi: 10.1104/pp.0 07781

Bañuelos, M. A., Klein, R. D., Alexander-Bowman, S. J., and Rodriguez-Navarro, A. (1995). A potassium transporter of the yeast Schwanniomyces occidentalis homologous to the Kup system of Escherichia coli has a high concentrative capacity. EMBO J. 14, 3021-3027.

Benito, B., Garciadeblas, B., and Rodriguez-Navarro, A. (2012). HAK transporters from Physcomitrella patens and Yarrowia lipolytica mediate sodium uptake. Plant Cell Physiol. 53, 1117-1123. doi: 10.1093/pcp/pcs056

Chen, G., Hu, Q., Luo, L., Yang, T., Zhang, S., Hu, Y., et al. (2015). Rice potassium transporter OsHAK1 is essential for maintaining potassium-mediated growth and functions in salt tolerance over low and high potassium concentration ranges. Plant Cell Environ. 38, 2747-2765. doi: 10.1111/pce.12585

Davies, C., Shin, R., Liu, W., Thomas, M. R., and Schachtman, D. P. (2006). Transporters expressed during grape berry (Vitis vinifera L.) development are associated with an increase in berry size and berry potassium accumulation. J. Exp. Bot. 57, 3209-3216. doi: 10.1093/jxb/erl091

Desbrosses, G., Kopka, C., Ott, T., and Udvardi, M. K. (2004). Lotus japonicus LjKUP is induced late during nodule development and encodes a potassium transporter of the plasma membrane. Mol. Plant Microbe Interact. 17, 789-797. doi: 10.1094/MPMI.2004.17.7.789

Elumalai, R. P., Nagpal, P., and Reed, J. W. (2002). A mutation in the Arabidopsis KT2/KUP2 potassium transporter gene affects shoot cell expansion. Plant Cell 14, 119-131. doi: 10.1105/tpc.010322

Evans, H. J., and Sorger, G. J. (1966). Role of mineral elements with emphasis on univalent cations. Annu. Rev. Plant Physiol. 17, 47-76. doi: 10.1146/annurev.pp.17.060166.000403

Fu, H. H., and Luan, S. (1998). AtKUP1: a dual-affinity $\mathrm{K}^{+}$transporter from Arabidopsis. Plant Cell 10, 63-73. doi: 10.2307/3870629

Garciadeblas, B., Barrero-Gil, J., Benito, B., and Rodriguez-Navarro, A. (2007). Potassium transport systems in the moss Physcomitrella patens: pphak1 plants reveal the complexity of potassium uptake. Plant J. 52, 1080-1093. doi: 10.1111/j.1365-313X.2007.03297.x

Garciadeblas, B., Benito, B., and Rodriguez-Navarro, A. (2002). Molecular cloning and functional expression in bacteria of the potassium transporters CnHAK1 and CnHAK2 of the seagrass Cymodocea nodosa. Plant Mol. Biol. 50, 623-633. doi: 10.1023/A:1019951023362

Gomez-Porras, J. L., Riaño-Pachón, D. M., Benito, B., Haro, R., Sklodowski, K., Rodríguez-Navarro, A., et al. (2012). Phylogenetic analysis of $\mathrm{K}^{+}$ transporters in bryophytes, lycophytes, and flowering plants indicates a specialization of vascular plants. Front. Plant Sci. 3:167. doi: 10.3389/fpls.2012. 00167

\section{SUPPLEMENTARY MATERIAL}

The Supplementary Material for this article can be found online at: http://journal.frontiersin.org/article/10.3389/fpls.2016.00127

TABLE S1 | List of HAK/KUP/KT sequences used in Figure 1 (Excel file).

Gupta, M., Qiu, X., Wang, L., Xie, W., Zhang, C., Xiong, L., et al. (2008) KT/HAK/KUP potassium transporters gene family and their whole-life cycle expression profile in rice (Oryza sativa). Mol. Genet. Genomics 280, 437-452. doi: 10.1007/s00438-008-0377-7

Jaquinod, M., Villiers, F., Kieffer-Jaquinod, S., Hugouvieux, V., Bruley, C., Garin, J., et al. (2007). A proteomics dissection of Arabidopsis thaliana vacuoles isolated from cell culture. Mol. Cell. Proteomics 6, 394-412. doi: 10.1074/mcp.M600250MCP200

Kim, E. J., Kwak, J. M., Uozumi, N., and Schroeder, J. I. (1998). AtKUP1: an Arabidopsis gene encoding high-affinity potassium transport activity. Plant Cell 10, 51-62. doi: 10.2307/3870628

Leigh, R. A., and Wyn Jones, R. G. (1984). A hypothesis relating critical potassium concentrations for growth to the distribution and functions of this ion in the plant cell. New Phytol. 97, 1-13. doi: 10.1111/j.1469-8137.1984.tb04103.x

Maathuis, F. J. M., and Sanders, D. (1994). Mechanism of high-affinity potassium uptake in roots of Arabidopsis thaliana. Proc. Natl. Acad. Sci. U.S.A. 91, 9272-9276. doi: 10.1073/pnas.91.20.9272

Marschner, P. (2012). Marschner's Mineral Nutrition of Higher Plants, 3rd Edn. London: Academic Press.

Martínez-Cordero, M. A., Martinez, V., and Rubio, F. (2004). Cloning and functional characterization of the high-affinity $\mathrm{K}^{+}$transporter HAK1 of pepper. Plant Mol. Biol. 56, 413-421. doi: 10.1007/s11103-004-3845-4

Mäser, P., Thomine, S., Schroeder, J. I., Ward, J. M., Hirschi, K., Sze, H., et al. (2001). Phylogenetic relationships within cation transporter families of Arabidopsis. Plant Physiol. 126, 1646-1667. doi: 10.1104/pp.126.4.1646

Nieves-Cordones, M., Aleman, F., Martinez, V., and Rubio, F. (2014). K+ uptake in plant roots. The systems involved, their regulation and parallels in other organisms. J. Plant Physiol. 171, 688-695. doi: 10.1016/j.jplph.2013.09.021

Nieves-Cordones, M., Martinez-Cordero, M. A., Martinez, V., and Rubio, F. (2007). An NH4+-sensitive component dominates high-affinity $\mathrm{K}^{+}$uptake in tomato plants. Plant Sci. 172, 273-280. doi: 10.1016/j.plantsci.2006.09.003

Osakabe, Y., Arinaga, N., Umezawa, T., Katsura, S., Nagamachi, K., Tanaka, H., et al. (2013). Osmotic stress responses and plant growth controlled by potassium transporters in Arabidopsis. Plant Cell 25, 609-624. doi: 10.1105/tpc.112. 105700

Qi, Z., Hampton, C. R., Shin, R., Barkla, B. J., White, P. J., and Schachtman, D. P. (2008). The high affinity $\mathrm{K}^{+}$transporter AtHAK5 plays a physiological role in planta at very low $\mathrm{K}^{+}$concentrations and provides a caesium uptake pathway in Arabidopsis. J. Exp. Bot. 59, 595-607. doi: 10.1093/jxb/erm330

Quintero, F. J., and Blatt, M. R. (1997). A new family of $\mathrm{K}^{+}$transporters from Arabidopsis that are conserved across phyla. FEBS Lett. 415, 206-211. doi: 10.1016/S0014-5793(97)01125-3

Ragel, P., Ródenas, R., García-Martín, E., Andrés, Z., Villalta, I., NievesCordones, M., et al. (2015). CIPK23 regulates HAK5-mediated highaffinity $\mathrm{K}^{+}$uptake in Arabidopsis roots. Plant Physiol. 169, 2863-2873. doi: 10.1104/pp.15.01401

Rigas, S., Debrosses, G., Haralampidis, K., Vicente-Agullo, F., Feldmann, K. A., Grabov, A., et al. (2001). TRH1 encodes a potassium transporter required for tip growth in Arabidopsis root hairs. Plant Cell 13, 139-151. doi: 10.1105/tpc.13.1.139

Rigas, S., Ditengou, F. A., Ljung, K., Daras, G., Tietz, O., Palme, K., et al. (2013). Root gravitropism and root hair development constitute coupled developmental responses regulated by auxin homeostasis in the Arabidopsis root apex. New Phytol. 197, 1130-1141. doi: 10.1111/nph.12092

Rodriguez-Navarro, A., Blatt, M. R., and Slayman, C. L. (1986). A potassiumproton symport in Neurospora crassa. J. Gen. Physiol. 87, 649-674. doi: 10.1085/jgp.87.5.649

Ruan, Y. L., Llewellyn, D. J., and Furbank, R. T. (2001). The control of single-celled cotton fiber elongation by developmentally reversible gating of plasmodesmata 
and coordinated expression of sucrose and $\mathrm{K}^{+}$transporters and expansin. Plant Cell 13, 47-60. doi: 10.1105/tpc.13.1.47

Rubio, F., Nieves-Cordones, M., Aleman, F., and Martinez, V. (2008). Relative contribution of AtHAK5 and AtAKT1 to $\mathrm{K}^{+}$uptake in the high-affinity range of concentrations. Physiol. Plant. 134, 598-608. doi: 10.1111/j.13993054.2008.01168.x

Rubio, F., Santa-Maria, G. E., and Rodriguez-Navarro, A. (2000). Cloning of Arabidopsis and barley cDNAs encoding HAK potassium transporters in root and shoot cells. Physiol. Plant. 109, 34-43. doi: 10.1034/j.13993054.2000.100106.x

Santa-María, G. E., Rubio, F., Dubcovsky, J., and Rodriguez-Navarro, A. (1997). The HAK1 gene of barley is a member of a large gene family and encodes a high-affinity potassium transporter. Plant Cell 9, 2281-2289. doi: 10.2307/38 70585

Scherzer, S., Böhm, J., Krol, E., Shabala, L., Kreuzer, I., Larisch, C., et al. (2015). Calcium sensor kinase activates potassium uptake systems in gland cells of Venus flytraps. Proc. Natl. Acad. Sci. U.S.A. 112, 7309-7314. doi: 10.1073/pnas.1507810112

Schleyer, M., and Bakker, E. P. (1993). Nucleotide sequence and 3'-end deletion studies indicate that the $\mathrm{K}(+)$-uptake protein kup from Escherichia coli is composed of a hydrophobic core linked to a large and partially essential hydrophilic C terminus. J. Bacteriol. 175, 6925-6931.

Senn, M. E., Rubio, F., Bañuelos, M. A., and Rodriguez-Navarro, A. (2001). Comparative functional features of plant potassium HvHAK1 and HvHAK2 transporters. J. Biol. Chem. 276, 44563-44569. doi: 10.1074/jbc.M1081 29200

Shen, Y., Shen, L., Shen, Z., Jing, W., Ge, H., Zhao, J., et al. (2015). The potassium transporter OsHAK21 functions in the maintenance of ion homeostasis and tolerance to salt stress in rice. Plant Cell Environ. 38, 2766-2779. doi: $10.1111 /$ pce. 12586
Song, Z. Z., Ma, R. J., and Yu, M. L. (2015). Genome-wide analysis and identification of KT/HAK/KUP potassium transporter gene family in peach (Prunus persica). Genet. Mol. Res. 14, 774-787. doi: 10.4238/2015.January.30.21

Very, A. A., Nieves-Cordones, M., Daly, M., Khan, I., Fizames, C., and Sentenac, H. (2014). Molecular biology of $\mathrm{K}^{+}$transport across the plant cell membrane: what do we learn from comparison between plant species? J. Plant Physiol. 171, 748-769. doi: 10.1016/j.jplph.2014.01.011

Vicente-Agullo, F., Rigas, S., Desbrosses, G., Dolan, L., Hatzopoulos, P., and Grabov, A. (2004). Potassium carrier TRH1 is required for auxin transport in Arabidopsis roots. Plant J. 40, 523-535. doi: 10.1111/j.1365-313X.2004. 02230.x

Wigoda, N., Moshelion, M., and Moran, N. (2014). Is the leaf bundle sheath a "smart flux valve" for $\mathrm{K}^{+}$nutrition? J. Plant Physiol. 171, 715-722. doi: 10.1016/j.jplph.2013.12.017

Yang, T., Zhang, S., Hu, Y., Wu, F., Hu, Q., Chen, G., et al. (2014). The role of a potassium transporter OsHAK5 in potassium acquisition and transport from roots to shoots in rice at low potassium supply levels. Plant Physiol. 166, 945-959. doi: 10.1104/pp.114.246520

Conflict of Interest Statement: The authors declare that the research was conducted in the absence of any commercial or financial relationships that could be construed as a potential conflict of interest.

Copyright (c) 2016 Nieves-Cordones, Ródenas, Chavanieu, Rivero, Martinez, Gaillard and Rubio. This is an open-access article distributed under the terms of the Creative Commons Attribution License (CC BY). The use, distribution or reproduction in other forums is permitted, provided the original author(s) or licensor are credited and that the original publication in this journal is cited, in accordance with accepted academic practice. No use, distribution or reproduction is permitted which does not comply with these terms. 